\title{
Erratum to: Effect of Surface Charge of Immortalized Mouse Cerebral Endothelial Cell Monolayer on Transport of Charged Solutes
}

\author{
Wei Yuan, ${ }^{1}$ Guanglei Li, ${ }^{1}$ Eun Seok Gil ${ }^{2}$ Tao Lu Lowe, ${ }^{2,3}$ and Bingmei M. Fu ${ }^{1}$
}

${ }^{1}$ Department of Biomedical Engineering, The City College of the City University of New York, 138th Street at Convent Avenue, New York, NY 10031, USA; ${ }^{2}$ Department of Surgery, Pennsylvania State University, Hershey, PA 17033, USA; and

${ }^{3}$ Department of Pharmaceutical Sciences, School of Pharmacy, Thomas Jefferson University, Philadelphia, PA 19107, USA

Erratum to: Annals of Biomedical Engineering (2010)

38(4):1463-1472, Epub 2010 Jan 20

DOI 10.1007/s10439-010-9920-x

Drs. Eun Seok Gil and Tao Lu Lowe were inadvertently omitted as authors of the publication. The author list appears correctly here.

Address correspondence to Bingmei M. Fu, Department of Biomedical Engineering, The City College of the City University of New York, 138th Street at Convent Avenue, New York, NY 10031, USA. Electronic mail: fu@ccny.cuny.edu

The online version of the original article can be found under doi: 10.1007/s10439-010-9920-x. 